\begin{tabular}{|c|c|c|c|}
\hline \multirow{2}{*}{$\begin{array}{r}\text { Case Reports in } \\
\text { Gastroenterology }\end{array}$} & \multicolumn{2}{|c|}{ Case Rep Gastroenterol 2017;11:329-334 } & \multirow[b]{2}{*}{$\begin{array}{l}\text { Karger } \\
\text { Open access }\end{array}$} \\
\hline & $\begin{array}{l}\text { DOI: 10.1159/000453659 } \\
\text { Publisned ontrne: TViay 19, } 2017\end{array}$ & $\begin{array}{l}\text { (c) } 2017 \text { The Author(s) } \\
\text { Published by S. Karger AG, Basel } \\
\text { www.karger.com/crg }\end{array}$ & \\
\hline & $\begin{array}{l}\text { This article is licensed under } \\
\text { International License (CC BY-I } \\
\text { Usage and distribution for comr }\end{array}$ & $\begin{array}{l}\text { nons Attribution-NonCommercia } \\
\text { ger.com/Services/OpenAccessLice } \\
\text { uires written permission. }\end{array}$ & \\
\hline
\end{tabular}

\title{
Fifteen-Year-Old Male with Type 2 Autoimmune Pancreatitis: An Argument for Endoscopy
}

\author{
Nathan T. Kolasinski $i^{a}$ Marc T. Johannsen ${ }^{b}$ Justin R. Hollon ${ }^{a}$ \\ ${ }^{a}$ Department of Pediatrics, Naval Medical Center Portsmouth, Portsmouth, VA, USA; \\ ${ }^{b}$ Department of Radiology, Naval Medical Center Portsmouth, Portsmouth, VA, USA
}

\section{Keywords}

Type 2 autoimmune pancreatitis · Inflammatory bowel disease · Endoscopy

\begin{abstract}
Type 2 autoimmune pancreatitis, an increasingly recognized etiology of pancreatitis in patients less than 20 years old, has characteristically been diagnosed with the histological finding of duct-centric pancreatitis in a patient who lacks elevated serum immunoglobulin G4. We present the case of a nonobese 15-year-old male, without any chronic medical conditions, who presented with the chief complaint of abdominal pain. The laboratory study results were remarkable for a lipase level of 5,419 U/L and a $\gamma$-glutamyl transferase level of 373 U/L. Magnetic resonance cholangiopancreatography revealed delayed contrast enhancement of the pancreas, diffuse parenchymal enlargement, and lack of normal lobulation. The patient's serum immunoglobulin G4 level was found to be $66 \mathrm{mg} / \mathrm{dL}$, which was within normal limits and supportive of a diagnosis of type 2 autoimmune pancreatitis. Despite the absence of intestinal complaints, the patient underwent subsequent endoscopy due to the correlation of type 2 autoimmune pancreatitis with inflammatory bowel disease that has been described in recent literature. Pan-colonic mild colitis was visualized, and the patient began treatment with steroids, to which he quickly responded. Performing endoscopy on this patient allowed for confident initiation of early therapy for both autoimmune pancreatitis and inflammatory bowel disease, and may have limited further surgical intervention and disease progression. For these reasons, this case highlights the utility of endoscopy in pediatric patients with suspected type 2 autoimmune pancreatitis, even in the absence of intestinal symptoms.
\end{abstract}




\section{Introduction}

Autoimmune pancreatitis (AIP) is a distinct form of pancreatitis that has become increasingly recognized in both adults and children. Classically, AIP has been characterized clinically by presentation with obstructive jaundice, histologically by a lymphoplasmacytic infiltrate and fibrosis, and therapeutically by a dramatic response to steroids. Imaging most typically reveals diffuse enlargement of the pancreas with delayed enhancement and loss of normal lobulation [1]. The pathogenic mechanisms of AIP are not completely understood, but, as with most autoimmune pathologies, AIP is thought to be the result of environmental exposures, i.e., immunogenic triggers, in genetically susceptible individuals [2]. As research has been conducted in Asia, Europe, and North America regarding this unique disease, clinical and histological profiling has revealed that AIP can be accurately divided into two distinct subtypes: type 1 and type $2[1,2]$.

Type 1 AIP, also known as lymphoplasmacytic sclerosing pancreatitis, occurs secondary to immunoglobulin (Ig) G4 systemic disease, with deposition of IgG4-positive plasma cells in the pancreas and other organs [1-3]. The most common presentation of type 1 AIP is painless jaundice in a middle-aged adult. There is a 3:1 male predominance, and type 1 AIP is frequently chronic [1,2]. Typically, type 1 AIP is diagnosed by observing 1 or more of the 5 cardinal features of AIP: typical imaging findings, elevated serum IgG4, other organ involvement, typical pancreatic histopathology, and response to steroid therapy [1]. The characteristic histopathologic pattern of type 1 AIP includes a dense lymphoplasmacytic infiltrate, a storiform pattern of fibrosis, and IgG4 immunostaining of the infiltrate revealing IgG4positive plasma cells accounting for $>40 \%$ of the total IgG-positive plasma cells [1-3].

Type 2 AIP, also known as idiopathic duct-centric pancreatitis and/or AIP with granulocytic epithelial lesions, is not associated with increased IgG4, is limited to the pancreas, and has characteristically been diagnosed with the histological finding of duct-centric pancreatitis [1-3]. Unlike type 1 AIP, the most common presentation of type 2 AIP is jaundice and acute pancreatitis in a patient less than 20 years old, and there is no sex bias [1,2]. The recent literature has noted a correlation between type 2 AIP and inflammatory bowel disease (IBD) [1-6]. Also in the literature, type 2 AIP has been referred to as less common that type 1 AIP. This is suspected, in part, to be due to challenges in obtaining a definitive diagnosis [1, 2]. Per the International Consensus Diagnostic Criteria for Autoimmune Pancreatitis, diagnosing "definitive type 2 AIP" requires pancreatic biopsy and the subsequent histologic finding of granulocytic infiltration of the duct wall with absent or scant IgG4-positive cells. However, in the absence of a pancreatic biopsy, a diagnosis of "probable type 2 AIP" relies on typical imaging findings in addition to a response to steroid therapy and coexisting IBD [1].

Our objective in presenting the following case is to demonstrate that upper and lower endoscopy should be considered for pediatric patients thought to have AIP, regardless of the presence or absence of intestinal symptoms. We argue that pursuing the diagnosis of "definitive type 2 AIP" in pediatric patients found to have coexisting IBD confers no clinical advantage or benefit once the diagnosis of "probable type 2 AIP" has been made.

\section{Clinical Case}

We present the case of a nonobese 15 -year-old male, without any chronic medical conditions, who presented with the chief complaint of abdominal pain. The abdominal pain had begun 10 days prior to presentation and was localized to the epigastric region. The patient 
described the pain as constant, cramp-like in quality, and nonradiating. After eating, the pain would escalate to an excruciating level and assume a stabbing quality severe enough to wake the patient from sleep if he went to bed soon after having a meal. The patient denied having diarrhea, hematochezia, and tenesmus. He had no history of recurrent abdominal pain, chronic weight loss, or growth stunting. A physical examination revealed an afebrile, uncomfortable, but non-toxic-appearing adolescent without jaundice or scleral icterus. The abdominal examination was significant for tenderness to palpation in the epigastric region without peritoneal signs.

The laboratory study results were remarkable for a lipase level of 5,419 U/L and a $\gamma$ glutamyl transferase level of $373 \mathrm{U} / \mathrm{L}$. Aspartate aminotransferase, alanine aminotransferase, alkaline phosphatase, and both total and direct bilirubin levels were all within normal limits. The patient did not have an elevated white blood cell count, nor was he anemic. Given the significantly elevated lipase level, imaging was ordered for further evaluation. Abdominal ultrasound was remarkable for common bile duct thickening and enlargement without evidence of cholelithiasis or choledocholithiasis; the pancreas was not visualized on this study. Subsequent magnetic resonance cholangiopancreatography (MRCP) revealed delayed contrast enhancement of the pancreas, diffuse parenchymal enlargement, and lack of normal lobulation (Fig. 1). This constellation of findings is referred to in the literature as a "sausage-like" pancreas [5]. The MRCP image also displayed biliary duct wall enhancement and dilation of the intrahepatic, extrahepatic, and common bile ducts; there was no noted stricture or narrowing.

As the MRCP findings were consistent with AIP, serum IgG4 levels were checked to delineate between type 1 and type 2 AIP; a serum IgG4 level $>150 \mathrm{mg} / \mathrm{dL}$ is consistent with type 1 AIP, whereas a level $<128 \mathrm{mg} / \mathrm{dL}$ is consistent with type 2 AIP [5]. The patient's serum IgG4 level was found to be $66 \mathrm{mg} / \mathrm{dL}$, which was within normal limits and supportive of a diagnosis of type 2 AIP. Due to the parenchymal imaging being consistent with AIP, and also due to concern for a biliary obstruction component, the patient was started on IV Solu-Medrol (20 mg, twice a day). A diagnostic biopsy of the pancreas was not performed. Within $24 \mathrm{~h}$, the patient's lipase level was $730 \mathrm{U} / \mathrm{L}$, and he was pain free and tolerating a regular diet.

Although the patient had no intestinal complaints, due to the noted correlation of type 2 AIP with IBD, the patient underwent subsequent endoscopy [2, 5, 6]. Pan-colonic mild colitis was visualized, with partial loss of vascularity, pinpoint ulcerations, mild mucosal friability, and fine granularity (Fig. 2). Biopsy specimens confirmed colitis (Fig. 3), and the patient was started on mesalamine (2.4 g, PO, twice a day) to treat his subclinical IBD. The diagnosis of IBD, along with the response to steroids, provided the collateral evidence to meet the diagnostic criteria for "probable type 2 AIP" [1]. At outpatient follow-up 20 days after having been started on glucocorticoids, the patient was asymptomatic of both abdominal pain and intestinal symptoms; he was tolerating a diet and his serum lipase level was $240 \mathrm{U} / \mathrm{L}$. The patient's pancreas was normal appearing on a repeat ultrasound performed 12 weeks after initiating glucocorticoid treatment.

\section{Discussion}

This case highlights the utility of endoscopy in pediatric patients with suspected type 2 AIP. Per the International Consensus Diagnostic Criteria for Autoimmune Pancreatitis, diagnosing "definitive type 2 AIP" requires pancreatic biopsy and the subsequent histologic find- 
ing of granulocytic infiltration of the duct wall (i.e., granulocytic epithelial lesions) with absent or scant $(<10$ cells/high-power field) IgG4-positive cells. However, in the absence of a pancreatic biopsy, a diagnosis of "probable type 2 AIP" relies on observing a "sausage-like" pancreas on imaging in addition to a response to steroid therapy and coexisting IBD [1].

In the case presented, the decision to proceed to endoscopy - despite no overt symptoms of IBD - was based solely upon the relatively high correlation of type 2 AIP with IBD [1-6]. With this patient, performing endoscopy allowed the identification and early treatment of subclinical IBD and, along with the patient's subsequent response to steroids, also provided the collateral evidence to meet the diagnostic criteria for "probable type 2 AIP" [1].

As pancreatic malignancy is exceedingly rare in the pediatric population, pancreatic biopsies are infrequently performed on children, and a logistical challenge exists in finding practitioners qualified to perform the procedure and obtain the necessary, adequate specimen [7]. Additionally, there may also be a delay in achieving a histologic examination, and there is a risk of inconclusive and/or false results $[2,8]$. Consequently, the focus on pancreatic histology in pediatric patients with suspected AIP may lead to delayed steroid treatment [6]. Endoscopic demonstration of IBD, on the other hand, allows for confident initiation of early therapy for both AIP and IBD, and may limit further surgical intervention and disease progression. For these reasons, we propose that endoscopy be considered in the initial evaluation of patients with suspected type 2 AIP.

\section{Statement of Ethics}

Informed consent was obtained from the appropriate party; a signed document is on file with the authors.

\section{Disclosure Statement}

We have no financial, professional, or personal conflicts of interests. The views expressed in this article are those of the authors and do not necessarily reflect the official policy or position of the Department of the Air Force, Department of the Navy, Department of Defense, or United States Government.

\section{Funding Sources}

We have not received any grants or outside funding. Specifically, we have not received any pharmaceutical or industry support.

\section{References}

1 Shimosegawa T, Chari ST, Frulloni L, Kamisawa T, Kawa S, Mino-Kenudson M, Kim MH, Klöppel G, Lerch MM, Löhr M, Notohara K, Okazaki K, Schneider A, Zhang L; International Association of Pancreatology: International consensus diagnostic criteria for autoimmune pancreatitis: guidelines of the International Association of Pancreatology. Pancreas 2011;40:352-358.

2 Hart PA, Zen Y, Chari ST: Recent advances in autoimmune pancreatitis. Gastroenterology 2015;149: 39-51. 
Kolasinski et al.: Fifteen-Year-Old Male with Type 2 Autoimmune Pancreatitis: An Argument for Endoscopy

-3 Chari ST, Klöppel G, Zhang L: Histopathologic and clinical subtypes of autoimmune pancreatitis: the Honolulu consensus document. Pancreatology 2010;10:664-672.

4 Okazaki K, Uchida K: Autoimmune pancreatitis: the past, present, and future. Pancreas 2015;44:10061016.

5 Patel Z, Patel S, Grendell J, Marciano T: Type 2 autoimmune pancreatitis: case report of a 9-year-old female and a review of the literature. Clin J Gastroenterol 2015;8:421-425.

-6 Lurz E, Gonska T: Pancreatic head mass leading to transient obstructive jaundice and diabetes mellitus in an adolescent. Gastroenterology 2015;149:e9-e10.

-7 Zen Y, Grammatikopoulos T, Hadzic N: Autoimmune pancreatitis in children: insights into the diagnostic challenge. J Pediatr Gastroenterol Nutr 2014;59:e42-e45.

-8 ASGE Standards of Practice Committee, Chandrasekhara V, Chathadi KV, Acosta RD, Decker GA, Early DS, Eloubeidi MA, Evans JA, Faulx AL, Fanelli RD, Fisher DA, Foley K, Fonkalsrud L, Hwang JH, Jue TL, Khashab MA, Lightdale JR, Muthusamy VR, Pasha SF, Saltzman JR, Sharaf R, Shaukat A, Shergill AK, Wang A, Cash BD, DeWitt JM: The role of endoscopy in benign pancreatic disease. Gastrointest Endosc 2015;82:203-214.

Copyright Statement: we are military service members. This work was prepared as part of our official duties. Title 17 U.S.C. 105 provides that "Copyright protection under this title is not available for any work of the United States Government." Title 17 U.S.C. 101 defines a United States Government work as a work prepared by a military service member or employee of the United States Government as part of that person's official duties.

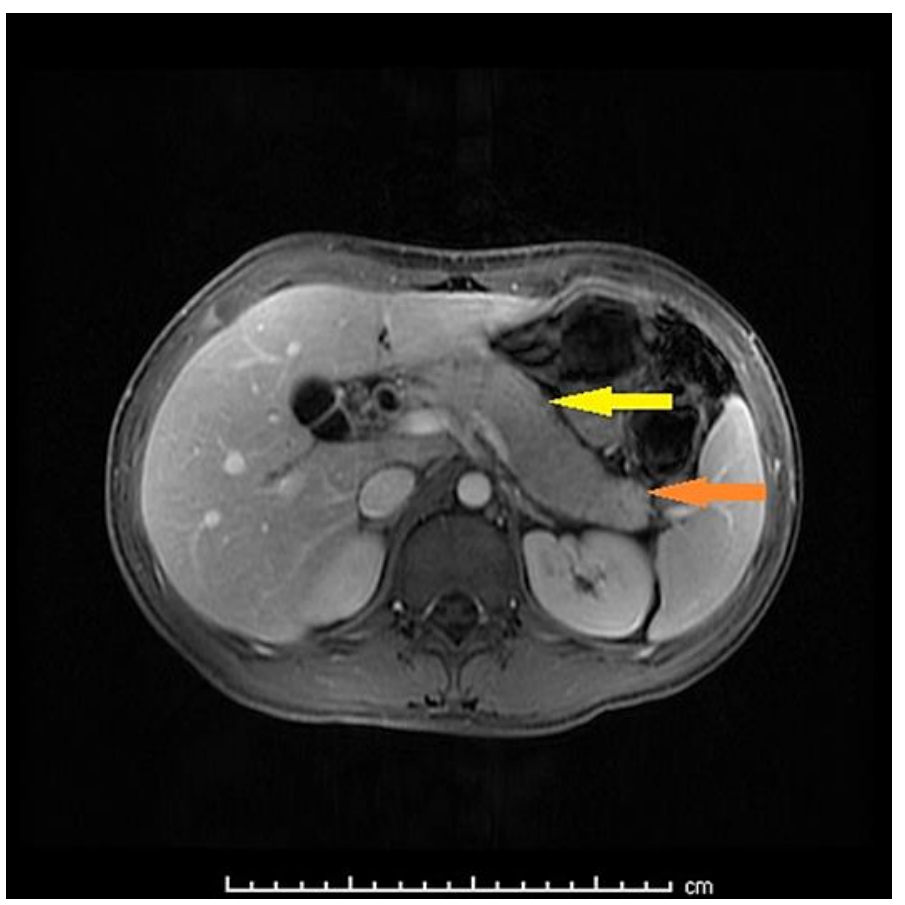

Fig. 1. Axial post-contrast T1-weighted image showing hypoenhancement and loss of normal lobulation of the body and head of the pancreas (yellow arrow) when compared to the tail of the pancreas (orange arrow). 


\section{Case Reports in Gastroenterology}

\begin{tabular}{l|l}
\hline Case Rep Gastroenterol 2017;11:329-334 \\
\hline DOI: 10.1159/000453659 & $\begin{array}{l}\text { ○ 2017 The Author(s). Published by S. Karger AG, Basel } \\
\text { www.karger.com/crg }\end{array}$ \\
\hline
\end{tabular}

Kolasinski et al.: Fifteen-Year-Old Male with Type 2 Autoimmune Pancreatitis: An Argument for Endoscopy

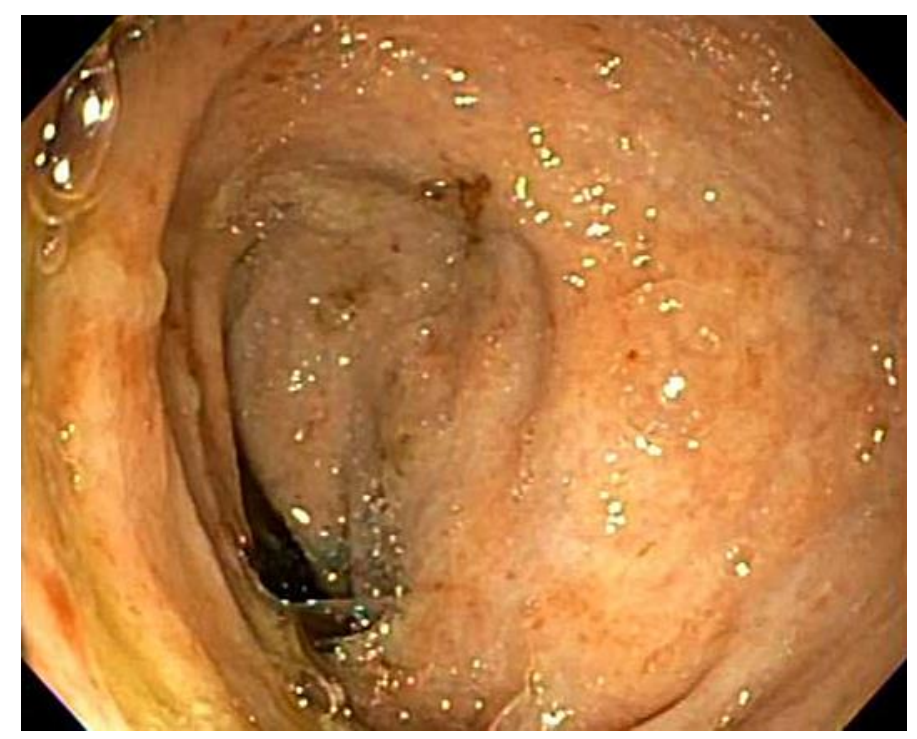

Fig. 2. Colonoscopy, descending colon, showing pan-colonic mild colitis with partial loss of vascularity, pinpoint ulcerations, mild mucosal friability, and fine granularity.

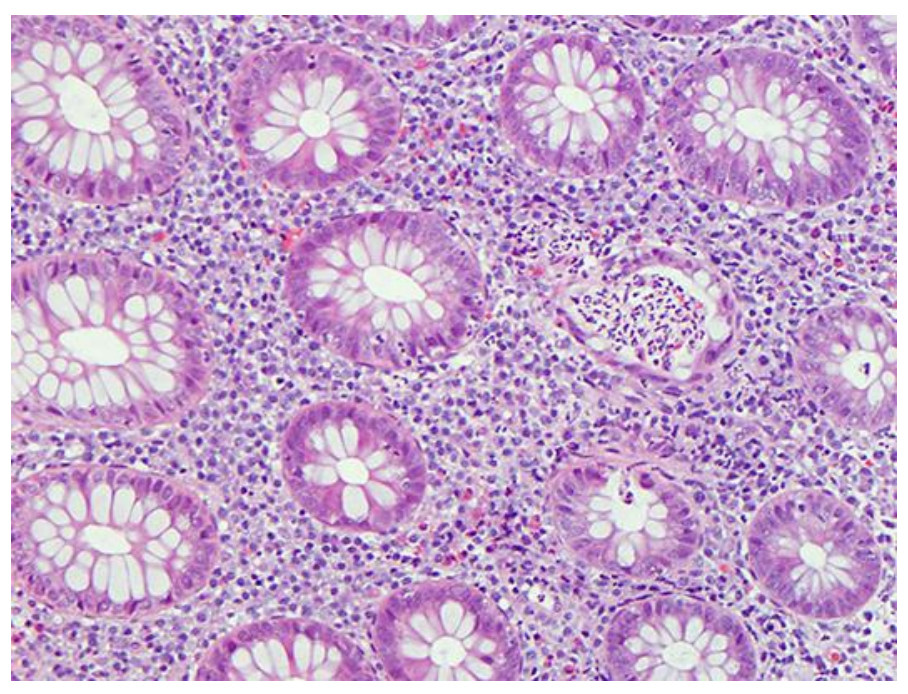

Fig. 3. Histopathology slide of the colon showing active cryptitis and crypt abscess. Original magnification, $\times 200$. 